\title{
Social Isolation During Postweaning Development Causes Hypoactivity of Neurons in the Medial Nucleus of the Male Rat Amygdala
}

\author{
Thomas Adams' and J Amiel Rosenkranz, I \\ 'Department of Cellular and Molecular Pharmacology, The Chicago Medical School, Rosalind Franklin University of Medicine and Science, \\ North Chicago, IL, USA
}

\begin{abstract}
Children exposed to neglect or social deprivation are at heightened risk for psychiatric disorders and abnormal social patterns as adults. There is also evidence that prepubertal neglect in children causes abnormal metabolic activity in several brain regions, including the amygdala area. The medial nucleus of the amygdala (MeA) is a key region for performance of social behaviors and still undergoes maturation during the periadolescent period. As such, the normal development of this region may be disrupted by social deprivation. In rodents, postweaning social isolation causes a range of deficits in sexual and agonistic behaviors that normally rely on the posterior MeA (MeAp). However, little is known about the effects of social isolation on the function of MeA neurons. In this study, we tested whether postweaning social isolation caused abnormal activity of MeA neurons. We found that postweaning social isolation caused a decrease of in vivo firing activity of MeAp neurons, and reduced drive from excitatory afferents. In vitro electrophysiological studies found that postweaning social isolation caused a presynaptic impairment of excitatory input to the dorsal MeAp, but a progressive postsynaptic reduction of membrane excitability in the ventral MeAp. These results demonstrate discrete, subnucleus-specific effects of social deprivation on the physiology of MeAp neurons. This pathophysiology may contribute to the disruption of social behavior after developmental social deprivation, and may be a novel target to facilitate the treatment of social disorders.

Neuropsychopharmacology (2016) 4I, 1929-1940; doi: I0.1038/npp.20I5.364; published online I3 January 2016
\end{abstract}

\section{INTRODUCTION}

The amygdala has a prominent role in mood and emotion. Intertwined with this role is the importance of the amygdala in social behavior. The amygdala is engaged by a range of social stimuli (Breiter et al, 1996; Fried et al, 1997, 2002; Davis et al, 2010), amygdala damage leads to impaired processing of social information (Adolphs et al, 1994, 2005), and patient populations with abnormal social behaviors often display abnormal amygdala activity (Baron-Cohen et al, 1999; Grady and Keightley, 2002; Stein et al, 2002; Wang et al, 2004; Williams et al, 2004; Phan et al, 2006; Coccaro et al, 2007; Rilling et al, 2007; Marsh et al, 2008; Jones et al, 2009; Kleinhans et al, 2009; Pinkham et al, 2011; Richey et al, 2014). For instance, patients with autism display hypoactivity of the amygdala during implicit processing of facial expressions (Critchley et al, 2000), and while detecting faces (Ashwin et al, 2006), and display hypoactivity or activity that does not parallel the task demands while making judgement

*Correspondence: Dr JA Rosenkranz, Department of Cellular and Molecular Pharmacology, The Chicago Medical School, Rosalind Franklin University of Medicine and Science, 3333 Green Bay Road, North Chicago, IL 60064, USA, Tel: + I 847578 8680, Fax: + I 847578 3268, E-mail: amiel.rosenkranz@rosalindfranklin.edu

Received 5 October 2015; revised 4 December 2015; accepted II December 2015; accepted article preview online 18 December 2015 of facial expressions (Baron-Cohen et al, 1999; Wang et al, 2004), although this may depend partly on attention towards faces and gaze direction (Weng et al, 2011; Tottenham et al, 2014). Furthermore, the activity of the amygdala is less coordinated with other brain regions involved in social behavior (Kleinhans et al, 2008; Rudie et al, 2012; von dem Hagen et al, 2013). The medial nucleus of the amygdala (MeA) has been particularly implicated in prosocial maternal and sexual behavior, as well as agonistic aggressive behaviors. In rodent models, the MeA is activated by socially relevant cues, such as maternal interaction, mating, pheromones of opposite-sex conspecifics, and predators (Fleming and Walsh, 1994; Fleming et al, 1994; Kollack-Walker and Newman, 1997; Meredith and Westberry, 2004; Choi et al, 2005; Samuelsen and Meredith, 2009b; Bergan et al, 2014), ablation of the MeA impairs recognition of opposite-sex odors (Petrulis, 2009; Maras and Petrulis, 2010), and activation of MeA afferents induces a gradient of social behavior from mating behavior to aggression (Hong et al, 2014; Unger et al, 2015).

Social experience during critical developmental periods is required for normal maturation of social behaviors (Einon et al, 1981; Potegal and Einon, 1989; Ikemoto and Panksepp, 1992; Hol et al, 1999; Pellis et al, 1999; van den Berg et al, 1999a, b; Von Frijtag et al, 2002). Rearing of rats in social isolation after weaning leads to a range of abnormal social 
behaviors including increased aggression, abnormal interactions with novel rodents, abnormal sexual behavior, and impaired social learning (Gerall et al, 1967; Meaney and Stewart, 1981; Hol et al, 1999; van den Berg et al, 1999a; Cooke et al, 2000; Von Frijtag et al, 2002; Melo et al, 2006; Agis-Balboa et al, 2007; Toth et al, 2012; Yusufishaq and Rosenkranz, 2013). Furthermore, postweaning social isolation causes reduced MeA volume (Cooke et al, 2000), reduced MeA neurogenesis (Lieberwirth et al, 2012), increased neural cell adhesion molecule (Gilabert-Juan et al, 2012), and morphological changes of MeA neurons (Ichikawa et al, 1993). However, it is not known whether social isolation after rearing leads to changes in the function of MeA neurons. The purpose of this study is to test whether postweaning social isolation perturbs the activity of MeA neurons.

The posterior MeA (MeAp) is particularly important in conspecific or socially relevant responses (Meredith and Westberry, 2004; Samuelsen and Meredith, 2009b), and was the focus of these studies. The MeAp has two functionally distinct subdivisions, the dorsal (MeApd) and ventral (MeApv) subdivisions. In this study, the in vivo activity of MeApd and MeApv neurons was measured from rats that were group housed or socially isolated in single housing beginning after weaning. Intrinsic neuronal excitability and excitatory synaptic drive are two key components that determine neuronal activity. Therefore, to determine whether the underlying proximal cause for altered MeAp neuronal activity was due to abnormal synaptic or intrinsic excitability, synaptic physiology and membrane excitability were measured in vitro. In addition, emergence of abnormal activity over the course of postweaning maturation could be due to interference with maturational processes or due to progressive impairment. Therefore, these alternatives were tested by measurement of the emergence of impairments over the course of social isolation.

\section{MATERIALS AND METHODS}

All procedures had prior approval from the Institutional Animal Care and Use Committee of Rosalind Franklin University, and followed the Guide for the Care and Use of Laboratory Animals (National Research Council, 2011). Care was taken to minimize animal distress and reduce the number of animals used. Male Sprague-Dawley rats (Harlan Laboratories, Indianapolis, IN) were weaned at postnatal day (P) 18, and upon arrival (P21-22) were housed at the Rosalind Franklin University animal facility (polycarbonate solid bottom cage, $43.2 \times 21.5 \times 20.3$ height, in $\mathrm{cm}$; Teklad Sani-Chips bedding). The rats were provided water and food (Rodent Diet 2020X pelleted feed, Harlan Teklad) ad libitum. The housing rooms were set to a $12 \mathrm{~h} / 12 \mathrm{~h}$ reverse light-dark cycle with lights off at 0700 hours. Temperature was maintained between 64 and $79^{\circ} \mathrm{F}$ and the humidity was maintained between 30 and $70 \%$. Rats were randomly assigned to control group housing (2-3/cage) or social isolation single housing (1/cage). All other aspects of husbandry were the same. Rats were used for experiments after 4-5 weeks (P50-60 postadolescents), or at intermediate time points, as described below. Several studies indicate that social behavior in males may be more sensitive to postweaning social isolation (Ferdman et al, 2007; Wall et al,
2012; Ahern et al, 2016). Therefore, males were the focus of the current study.

\section{Experimental Outline}

Rats were group housed our single housed on the day of arrival, and then tested at noted timepoints. To test the effects of social isolation on MeA activity, in vivo electrophysiological measures were obtained from young adults (after more than 4 weeks; P50-60). Both single-unit (group housing $n=9$ rats; social isolation single housing $n=10$ rats) and evoked field potentials were measured from MeApd and MeApv (MeApd group housing $n=9$ rats, social isolation single housing $n=8$ rats; MeApv group housing, $n=9$ rats, social isolation single housing, $n=7$ rats). To test the proximal causes for a change in MeA activity, in vitro whole-cell recordings were also obtained from young adults to measure synaptic activity (8 rats per group) and membrane responsiveness (8 rats per group). To determine the time course of the changes over the postweaning period, in vitro whole-cell recordings were obtained after 1 day (P22-23, $n=8$ rats per group), 1 week (P28-29, group housing, $n=8$ rats, single housing, $n=7$ rats), 2 weeks (P35-36, group housing, $n=7$ rats, single housing, $n=7$ rats), or as young adults (P50-60, $n=8$ rats per group).

\section{In vivo Electrophysiology}

Rats were anesthetized with urethane $(1.5 \mathrm{~g} / \mathrm{kg}$, intraperitoneally) and $2 \%$ lidocaine jelly was infiltrated into their ears. Rats were placed in a stereotaxic apparatus (David Kopf Instruments, Tujunga, CA or Stoelting Instruments, Wood Dale, IL), and core body temperature was maintained at $\sim 37^{\circ} \mathrm{C}$ (TC-1000 Temperature Controller; CWE, Ardmore, PA). Bore holes were drilled in the skull overlying the lateral nucleus of the amygdala (LAT; centered on $-5.0 \mathrm{~mm} \mathrm{M}-\mathrm{L}$, $-3.0 \mathrm{~mm} \mathrm{A-P}$ from bregma) and the MeA (centered on $-3.2 \mathrm{~mm} \mathrm{M}-\mathrm{L},-3.3 \mathrm{~mm} \mathrm{~A}-\mathrm{P}$ from bregma, between 8.0 and $9.5 \mathrm{~mm} \mathrm{D}-\mathrm{V})$. A concentric bipolar stimulation electrode (Rhodes Medical Instrument, Summerland, CA) was slowly lowered into the LAT to deliver stimulation and to record spontaneous local field potentials as a gauge of anesthesia state. After a minimum of $45 \mathrm{~min}$, a glass microelectrode (2.0 mm outer diameter borosilicate; A-M Systems, Carlsborg, WA) was pulled (PE-2 microelectrode puller; Narishige Group, Tokyo, Japan) and filled with $2 \%$ Pontamine Sky Blue (Alfa Aesar, Ward Hill, MA) in $2 \mathrm{M} \mathrm{NaCl}$ (Thermo Fisher Scientific, Waltham, MA). The glass electrode was slowly lowered to the MeA to record neuronal activity. Signals were amplified (2400 Extracellular Preamplifier; Dagan, Minneapolis, MN) and digitized (5-10 kHz; InstruTECH ITC-18; HEKA Instruments, Bellmore, NY). Signals were monitored audially (AM10 amplifier; Grass Technologies, Warwick, RI) and visually (AxoGraph X software version 1.3.5; Axograph Scientific, Sydney, Australia) and saved for later analysis (Mac Pro; Apple, Cupertino, CA). Electrical stimulation was delivered (S88 Stimulator and PSIU6 Stimulation Isolation Unit; Grass Technologies, Warwick, RI) through the bipolar electrode with an intensity range of $0.1-0.9 \mathrm{~mA}, 0.2 \mathrm{~ms}$ duration, repeating at $0.2-40 \mathrm{~Hz}$.

At the conclusion of experiments, Pontamine Sky Blue was iontophoresed through the recording electrode $(-30 \mu \mathrm{A}$, 
$>30$ min; Constant Current Source, Fintronics, Orange, CT). The brain was removed and placed in $4 \%$ paraformaldehyde in $0.1 \mathrm{M}$ phosphate buffer overnight, and then transferred to $25 \%$ sucrose (Thermo Fisher Scientific, Bannockburn, IL) in $0.1 \mathrm{M}$ phosphate buffer. Brains were sectioned $(60 \mu \mathrm{m}$ thick) with a freezing microtome (Leica Microsystems, Buffalo Grove, IL) and stained with cresyl violet (Sigma-Aldrich, St Louis, MO). Recording and stimulation sites were verified by light microscopy.

\section{In vitro Electrophysiology}

Rats were deeply anesthetized with a mixture of ketamine (80-100 mg/kg; Webster Veterinary Supply, Devens, MA) and xylazine (10-20 mg/kg; Webster Veterinary Supply). Upon verification of deep anesthesia by the absence of response to foot pinch, rats were decapitated, and brains were rapidly immersed in ice-cold, aerated $\left(95 \% \mathrm{O}_{2} / 5 \%\right.$ $\mathrm{CO}_{2}$ ) high sucrose artificial cerebrospinal fluid (ACSF) containing (in mM) $2.5 \mathrm{KCl}, 1.25 \mathrm{NaH}_{2} \mathrm{PO}_{4}, 25 \mathrm{NaHCO}_{3}$, 7 dextrose, $7 \mathrm{MgCl}_{2}, 0.5 \mathrm{CaCl}_{2}, 210$ sucrose, 1.3 ascorbic acid, 3 sodium pyruvate, and $\sim 290 \mathrm{mOsm}$. The brain was sectioned at 250-300 $\mu \mathrm{m}$ (Vibratome 1000; Ted Pella, Redding, CA) in the same ACSF. Brain sections were then placed in physiological ACSF saturated with 95\% $\mathrm{O}_{2} / 5 \%$ $\mathrm{CO}_{2}$, containing (in mM) $125 \mathrm{NaCl}, 2.5 \mathrm{KCl}, 1.25 \mathrm{NaH}_{2} \mathrm{PO}_{4}$, $25 \mathrm{NaHCO} 3,10$ dextrose, $1 \mathrm{MgCl}_{2}$, and $2 \mathrm{CaCl}_{2}$, with the addition of $1.3 \mathrm{mM}$ ascorbic acid and $3 \mathrm{mM}$ sodium pyruvate for $50-60 \mathrm{~min}$ at $34^{\circ} \mathrm{C}$. Recordings were performed at $32-$ $34^{\circ} \mathrm{C}$ in submerged slices in physiological ACSF (as above, without ascorbic acid or sodium pyruvate). When specified, (+)-bicuculline $(10 \mu \mathrm{M}$; Ascent Scientific, Princeton, NJ; dissolved in dimethyl sulfoxide), picrotoxin $(10 \mu \mathrm{M}$; SigmaAldrich; dissolved in ethanol), 6-cyano-7-nitroquinoxaline2,3-dion (CNQX) disodium salt $(10 \mu \mathrm{M}$; Ascent Scientific; dissolved in $\mathrm{ddH}_{2} \mathrm{O}$ ), and DL-2-amino-5-phosphonopentanoic acid sodium salt $(50 \mu \mathrm{M} ;$ Abcam Biochemicals, Cambridge, MA; dissolved in $100 \mathrm{mM} \mathrm{NaOH}$ ) were added to the ACSF to block GABAA-, AMPA- and NMDA receptor-mediated currents. Final solvent concentrations were $<0.1 \%$ of the total ACSF volume.

Recording pipettes were pulled (borosilicate glass, $2.0 \mathrm{~mm}$ outer diameter, 2-8 $\mathrm{M} \Omega$ open tip resistance; Sutter Instruments, Novato, CA). Electrodes were filled with (in mM) 120 K-gluconate, $20 \mathrm{KCl}, 0.2 \mathrm{EGTA}, 10 \mathrm{HEPES}, 2 \mathrm{NaCl}, 4 \mathrm{ATP}$ $\mathrm{Mg}, \quad 0.3$ GTP-Tris, 7 Tris-phosphocreatine, and $0.2 \%$ neurobiotin (Vector Laboratories, Burlingame, CA), with a $\mathrm{pH}$ of 7.3. Whole-cell recordings were performed from visualized neurons in the MeAp under IR-DIC conditions. Signals were amplified (AxoClamp 2B; Molecular Devices, Sunnyvale, CA) and low-pass-filtered at $3-5 \mathrm{kHz}$ and digitalized at $10 \mathrm{kHz}$ (InstruTECH ITC-18; HEKA Instruments). Mean series resistance for each group was below $25 \mathrm{M} \Omega$. Data were monitored and saved for later analysis (AxoGraph X software v.1.3.5 (Axograph Scientific), on Mac Pro (Apple)). Upon completion of recordings, slices were fixed overnight and stored for up to 3 weeks at $4{ }^{\circ} \mathrm{C}(4 \%$ paraformaldehyde in $0.1 \mathrm{M}$ phosphate-buffered saline, PBS). Sections were rinsed three times with PBS, treated with Triton X-100 (VWR International, Radnor, PA; 1\% in PBS) for 6 to $8 \mathrm{~h}$, and then incubated in the Vectastain $A B C$ Reagent (Vector Laboratories) in PBS at room temperature overnight. After three rinses with PBS, sections were reacted with diaminobenzidine (DAB) and $\mathrm{H}_{2} \mathrm{O}_{2}$ (Peroxidase Substrate Kit DAB; Vector Laboratories) in water to visualize the neurobiotin-filled neurons. Sections were washed in PBS repeatedly to stop the reaction. Sections were mounted, dried, and coverslipped. Stained sections were used to localize the recording sites.

\section{Data Analysis and Statistics}

Recording sites in vivo and in vitro that were confirmed to lie within the MeAp were analyzed. MeApd and MeApv subdivisions were delineated based on the atlas of Paxinos and Watson (Paxinos, 2009). In vivo firing rate was quantified as the number of extracellular action potentials per $\mathrm{s}$ over at least $5 \mathrm{~min}$ time epochs. Extracellular action potentials required a signal-to-noise ratio of at least 3:1, consistent amplitude, and half-width $(<15 \%$ change over $5 \mathrm{~min}$ ) for inclusion in analysis. Evoked field potentials were measured upon stimulation of the LAT $(0.2 \mathrm{~Hz}, 0.1 \mathrm{~ms}$ duration). When LAT train stimulation was examined $(10-40 \mathrm{~Hz})$, a stimulation intensity to evoke a half-maximal response was used, with an intertrain interval of $30 \mathrm{~s}$. A minimum of 10 sweeps was averaged and the slope of the field potential was measured. Excitatory postsynaptic currents (EPSCs) were measured in vitro with whole-cell recordings in the presence of GABA and NMDA receptor blockers (above). A bipolar stimulation electrode was placed $\sim 100 \mu \mathrm{m}$ from the soma of the recorded neuron to evoke excitatory postsynaptic responses $(0.2 \mathrm{~Hz}, 0.1 \mathrm{~ms}$ duration, intensity to evoke 30-80 pA EPSC). A minimum of 20 sweeps was averaged and the peak amplitude of the EPSC was measured. Miniature excitatory postsynaptic currents (mEPSCs) were measured in the presence of GABA blockers (above) and TTX $(1 \mu \mathrm{M})$ and quantified using a variable amplitude template (Axograph $\mathrm{X}$ ), with a required signal-tonoise ratio of three times the standard deviation of the noise. Frequency, amplitude, half-width, and decay were quantified. Resting membrane potential $\left(V_{\text {rest }}\right)$ was measured as the membrane voltage in the absence of holding current in the voltage clamp. In separate experiments, GABA receptor blockers (above) and glutamate receptor (NMDA and AMPA) blockers were applied and subsequent whole-cell voltage measures were obtained in bridge mode. In these experiments, $V_{\text {rest }}$ was measured as the membrane voltage in the absence of current. The remainder of the voltage recordings were obtained while holding the membrane potential close to $-70 \mathrm{mV}$. Input resistance $\left(R_{\mathrm{n}}\right)$ was measured as the linear fit of the voltage response to hyperpolarizing current steps ( -10 to $-40 \mathrm{pA})$. Membrane excitability was measured as the number of action potentials evoked by depolarizing current steps (20-80 pA). This measure was best fit to a second-order polynomial to derive the current amplitude that evokes a $50 \%$ response $\left(\mathrm{EC}_{50}\right)$ and the extrapolated maximal number of action potentials $\left(B_{\max }\right)$. Neurons that displayed electrophysiological parameters that match GABAergic interneurons of the MeA (Keshavarzi et al, 2014) or displayed $>15 \%$ variability across time in holding current, $V_{\text {rest }}$, input resistance or access resistance were not included for analysis. Data were analyzed using Prism (version 6.0; GraphPad Software, La Jolla, CA), with a $p<0.05$ considered statistically significant. Data were 
tested for normal distribution (D'Agostino and Pearson's normality test) and homogeneity of variance (Bartlett's test). Planned comparisons across one factor were performed with a two-tailed unpaired $t$-test. If more than two groups were compared, a one-way ANOVA was used instead. When multiple factors were compared between groups, a two-way ANOVA (or if applicable, two-way repeated-measures (RM)-ANOVA) was used. ANOVAs were followed with comparisons between individual groups using Holm-Sidak's multiple comparisons tests. Data are presented as mean \pm SEM, unless noted otherwise.

\section{RESULTS}

The firing rate of $\mathrm{MeA}$ neurons was measured after $>4$ weeks of control group housing or social isolation single housing ( $N=9-10$ rats per group, see Materials and Methods section). Neurons were histologically confirmed to lie within the MeApd or MeApv (Figure 1a). Social isolation significantly decreased the in vivo firing rate of MeA neurons (Figure 1b; control $3.0 \pm 0.4 \mathrm{~Hz}, n=33$ neurons, social isolation $1.3 \pm 0.2 \mathrm{~Hz}, n=35$ neurons; $p=0.0004, t=3.74$, two-tailed unpaired $t$-test). This decreased neuronal firing was observed in both MeApd (Figure 1c; $p=0.048, t=2.07$, d.f. $=30$; control $3.6 \pm 0.7 \mathrm{~Hz}, n=17$ neurons, social isolation $1.9 \pm 0.4 \mathrm{~Hz}, n=15$ neurons) and MeApv (Figure 1c; $p=0.0008, t=3.70$; control $2.7 \pm 0.5 \mathrm{~Hz}, n=16$ neurons, social isolation: $0.9 \pm 0.2 \mathrm{~Hz}, n=20$ neurons). The firing of MeA neurons can be driven by their response to excitatory inputs. MeA receives excitatory glutamatergic inputs from several regions, including the LAT (Pitkanen et al, 1995). LAT was chosen for stimulation because its output is glutamatergic (McDonald, 1996) and it has functional importance in guidance of affective behavior, including some social behaviors (Hoffman et al, 2007; Davis et al, 2010; Jeon et al, 2010; Gadziola et al, 2012). LAT stimulation evoked a stimulation intensity-dependent local field potential in the MeA (Figure 1d; slope of the local field potential across intensity, main effect of intensity $p<0.0001, F(4,124)=$ 360.4, two-way RM-ANOVA). Social isolation significantly decreased the response of the MeA to LAT stimulation (Figure 1d; main effect of social isolation $p=0.001$, $\mathrm{F}(1,31)=13.36$, social isolation $\times$ intensity interaction $p<0.0001, \mathrm{~F}(4,124)=15.55$, two-way RM-ANOVA, $N=9$ rats per group; $p<0.05$ at $0.5-0.9 \mathrm{~mA}$, Holm-Sidak's multiple comparisons test). The effect of social isolation on evoked excitatory input was specific for MeApd (Figure 1e; social isolation $\times$ intensity interaction $p<0.0001, \mathrm{~F}(4,60)=18.47$, control $n=9$ rats, social isolation $n=8$ rats) and not statistically significant in MeApv (Figure 1e; social isolation $\times$ intensity interaction $p=0.081, F(4,56)=2.19$, two-way RM-ANOVA, control $n=9$ rats, social isolation $n=7$ rats). This indicates that, while social isolation has similar overall effects on the firing of MeApd and MeApv neurons, different mechanisms may underlie these effects in MeApd and MeApv.

Several mechanisms can cause decreased firing and response to excitatory afferents, including presynaptic and postsynaptic factors. To test the proximal cause of the effect of social isolation, we first measured postsynaptic aspects that influence neuronal responsiveness while synaptic inputs a
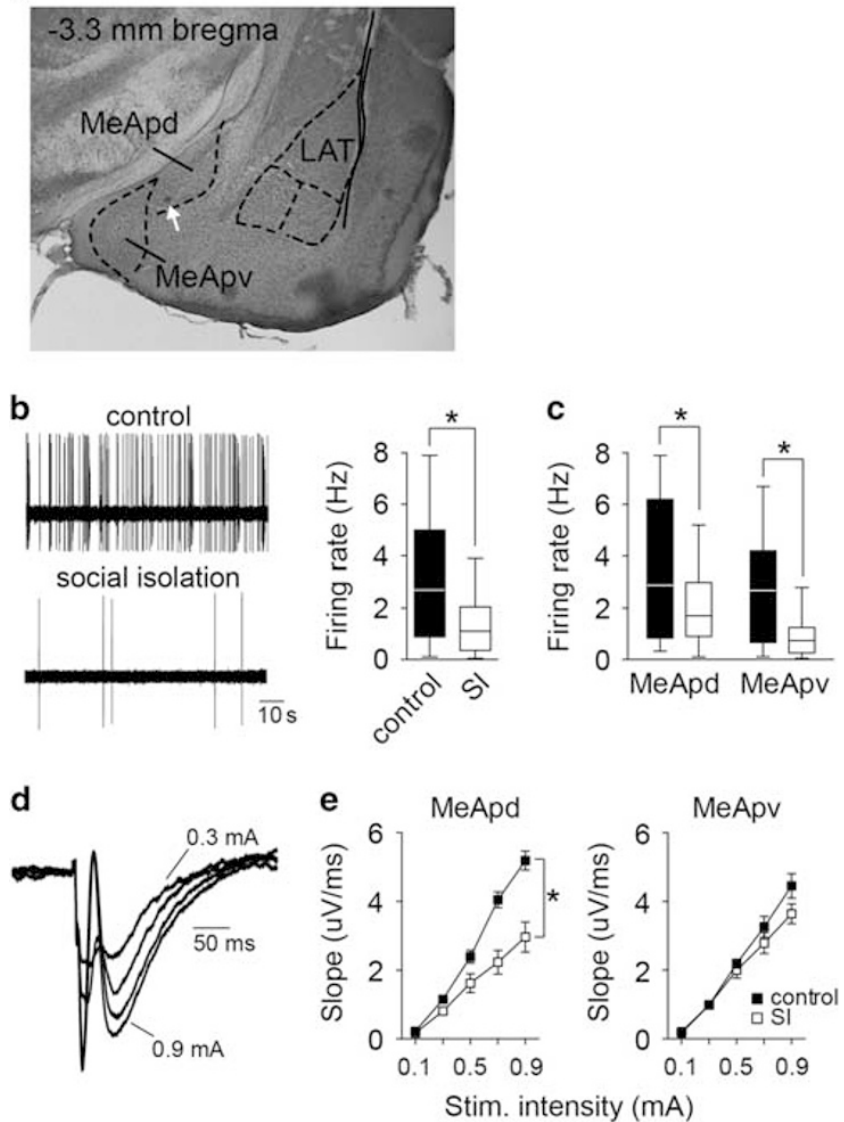

Figure I Postweaning social isolation decreases the in vivo activity of medial nucleus of the amygdala (MeA) neurons. (a) Recording sites in MeApd (MeA dorsal) and MeApv (MeA ventral) neurons. MeApd and MeApv were histologically verified by the deposit of Pontamine Sky Blue (white arrow). The section displayed here corresponds to $-3.30 \mathrm{~mm}$ caudal from bregma in the atlas of Paxinos, 2009. (b) In vivo extracellular recordings (left) were obtained from control group-housed or age-matched isolationreared rats. The firing rate of $\mathrm{MeA}$ neurons from isolation-reared rats was significantly less than group-housed rats (box plot \pm Tukey). $* p<0.05$ twotailed unpaired t-test. (c) The firing rate of MeApd and MeApv neurons from isolation-reared rats was significantly less than group-housed rats. $* P<0.05$ in post hoc Holm-Sidak's multiple comparisons test after two-way repeatedmeasures analysis of variance (ANOVA). (d) Field potentials evoked by stimulation of lateral nucleus of the amygdala (LAT) displayed stimulation intensity-dependent amplitude (left). (e) The slope of the evoked field potentials was significantly lower in the isolation-reared group in MeApd (left) but not MeApv (right). $* P<0.05$ two-way repeatedmeasures ANOVA.

were blocked in vitro (see Materials and Methods section, $n=8$ rats per group). There was no significant difference in the resting membrane potential between control and social isolation groups (Figure 2a; $V_{\text {rest }}$ control $-60.6 \pm 0.6 \mathrm{mV}$, $n=24$ neurons, social isolation $-61.9 \pm 0.7 \mathrm{mV}, n=27$ neurons, $p=0.182, t=1.364$, d.f. $=34$, two-tailed unpaired $t$-test). Input resistance was measured to gauge neuronal membrane responsiveness (Figure $2 \mathrm{~b}$ ). Social isolation significantly decreased input resistance (control 206.9 \pm $15.1 \mathrm{M} \Omega, n=18$ neurons, social isolation $151.9 \pm 13.1 \mathrm{M} \Omega$, $n=18$ neurons, $p=0.009, t=2.76$, d.f. $=34$, two-tailed unpaired $t$-test). However, these effects of social isolation were nucleus-specific, with reduced input resistance of 


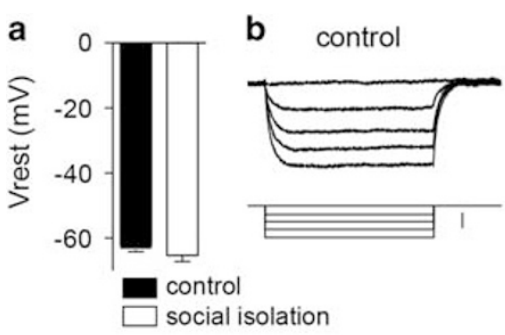

d

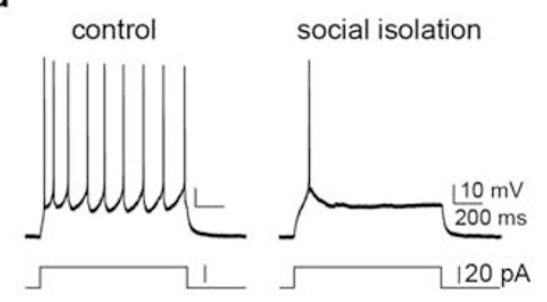

social isolation

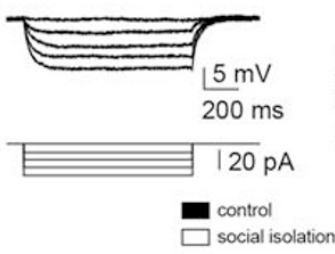

e

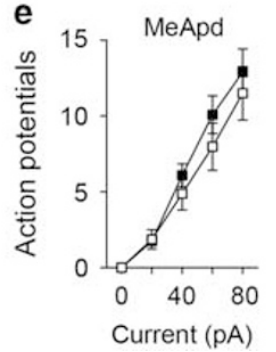

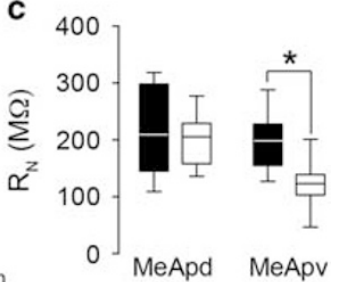

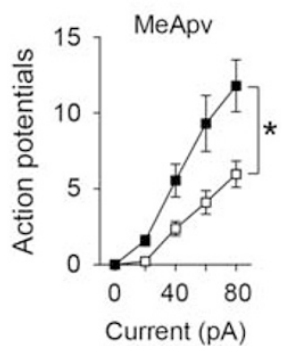

Figure 2 Postweaning social isolation decreases membrane excitability of medial nucleus of the amygdala (MeA) neurons. MeA neurons were recorded in vitro using whole-cell recordings. Blockers of $\gamma$-aminobutyric acid (GABA) and glutamate were present during recordings. (a) The resting membrane potential was not significantly different between group-housed and isolation-reared rats. (b) Input resistance was measured as the voltage response to hyperpolarizing current steps (left). (c) Social isolation decreased the input resistance in neurons from the MeApv (MeA ventral) but not the MeApd (MeA dorsal) neurons (box plot \pm Tukey). ${ }^{*} P<0.05$ in post hoc Holm-Sidak's multiple comparisons test after two-way repeated-measures analysis of variance (ANOVA). (d) Membrane excitability was quantified as the number of action potentials in response to depolarizing current steps (left). (e) Social isolation decreased membrane excitability in neurons from the MeApv but not the MeApd. $* P<0.05$ two-way repeated-measures ANOVA.

MeApv neurons but not MeApd neurons (Figure 2c; social isolation $\times$ nucleus interaction $p=0.037, \quad \mathrm{~F}(1,32)=4.738$; Holm-Sidak's multiple comparisons test MeApd $p>0.05$, control $n=9$ neurons, social isolation $n=7$ neurons, MeApv $p<0.05$ (control $n=9$ neurons, social isolation $n=11$ neurons)).

Membrane excitability, measured as action potential firing in response to depolarization (from $-70 \mathrm{mV}$; Figure 2d), was significantly decreased after social isolation (main effect of social isolation $p<0.0001, F(4,112)=60.21$; social isolation $\times$ current step amplitude interaction $p<0.0001$, $\mathrm{F}(4,112)=10.55$, two-way RM-ANOVA; significant differences at $40-80 \mathrm{pA}$ current steps, $p<0.05$ Holm-Sidak's multiple comparisons test). These effects of social isolation were similarly nucleus-specific, with reduced membrane excitability only in MeApv neurons (Figure 2e; main effect of social isolation $p=0.0049, \mathrm{~F}(1,25)=9.516$; social isolation $\times$ current step amplitude interaction $p<0.0001$, $\mathrm{F}(4,100)=6.676$; Holm-Sidak's multiple comparisons test $p<0.05,40-80 \mathrm{pA}, n=13$ control neurons, $n=14$ social isolation neurons), and not MeApd neurons (main effect of social isolation $p=0.47, \mathrm{~F}(1,22)=0.551$; social isolation $\times$ current step amplitude interaction $p=0.523, \mathrm{~F}(4,88)=0.809$; $n=11$ control neurons, $n=13$ social isolation neurons). This demonstrates a postsynaptic reduction of neuronal excitability that can contribute to decreased firing of MeApv neurons.

Neuronal activity can be driven by excitatory synaptic inputs. Therefore, presynaptic factors that influence the release of glutamate can also significantly impact neuronal activity. To test if social isolation decreases the strength of glutamatergic inputs, glutamatergic synaptic currents were measured in vitro (Figure 3a; $n=9$ rats per group, see Materials and Methods section). The frequency of mEPSCs was significantly lower after social isolation (Figure 3a; control $3.9 \pm 0.5 \mathrm{~Hz}, \quad n=22$ neurons, social isolation $2.0 \pm 0.3 \mathrm{~Hz}, n=20$ neurons, $p=0.0069, t=2.847$, two-tailed unpaired $t$-test). Social isolation decreased mEPSC frequency recorded from MeApd but not MeApv neurons (Figure 3b; nucleus $\times$ social isolation interaction, $p=0.049, \mathrm{~F}(1,38)=4.133$, two-way ANOVA; Holm-Sidak's multiple comparisons test, MeApd $p<0.05$, control $n=12$ neurons, social isolation $n=10$ neurons; Holm-Sidak's multiple comparisons test, MeApv $p>0.05$, control $n=10$ neurons, social isolation $n=10$ neurons). There was no significant effect of social isolation on mEPSC amplitude (Figure 3c; control $10.5 \pm 0.4 \mathrm{pA}, n=22$ neurons, social isolation $9.7 \pm 0.6 \mathrm{pA}, n=20$ neurons, $p=0.246$, $t=1.177$, two-tailed unpaired $t$-test) and no significant difference in mEPSC decay time (control $4.3 \pm 0.3 \mathrm{~ms}, n=22$ neurons, social isolation $4.1 \pm 0.3 \mathrm{~ms}, n=20$ neurons; $p=0.81$, $t=0.237$, two-tailed unpaired $t$-test; control $4.3 \pm 0.3 \mathrm{~ms}, n=22$ neurons, social isolation $4.2 \pm 0.3 \mathrm{~ms}, n=20$ neurons). Pairedpulse ratio was measured as an additional index of presynaptic function. Social isolation caused a change in paired-pulse response, from slight facilitation towards depression (Figure 3d; $p=0.006, t=2.944$, d.f. $=35$, two-tailed unpaired $t$-test; control $n=18$, social isolation $n=19$ ), but this was specific for the paired-pulse response of MeApd neurons but not MeApv neurons (Figure 3d; nucleus $\times$ social isolation interaction, $p=0.032, \mathrm{~F}(1,34)=5.035$, two-way ANOVA; Holm-Sidak's multiple comparisons test, MeApd $p<0.05$, control $n=8$ neurons, social isolation $n=9$ neurons; Holm-Sidak's multiple comparisons test, MeApv $p>0.05$, control $n=10$ neurons, social isolation $n=11$ neurons). This demonstrates that social isolation modifies presynaptic factors in MeApd but not MeApv neurons.

These data indicate that presynaptic and postsynaptic factors can contribute to in vivo impairments of the MeA 
a

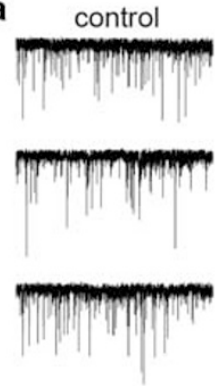

C

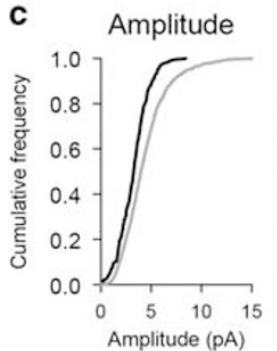

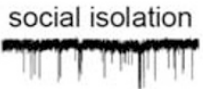
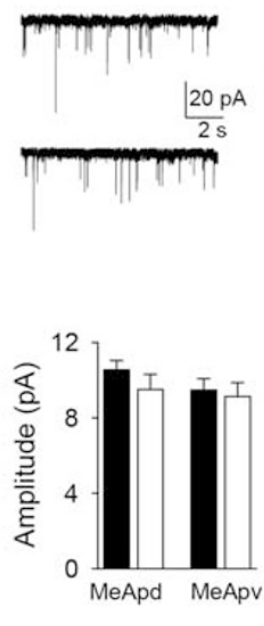

Frequency

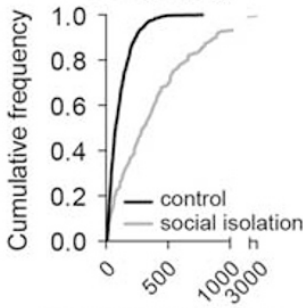

Interevent interval (ms)

d

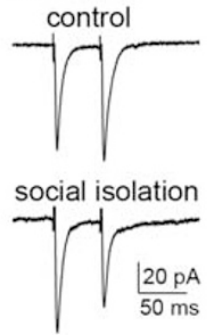

b

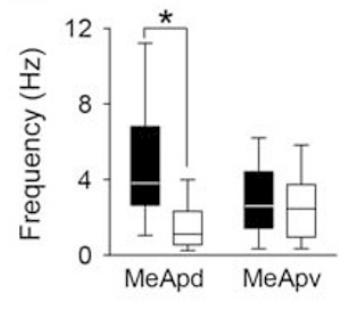

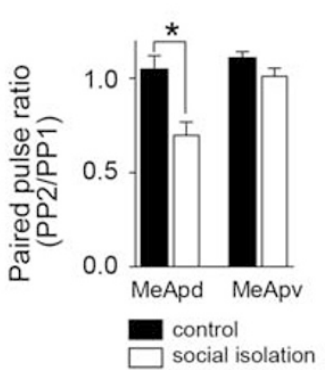

Figure 3 Postweaning social isolation decreases excitatory synaptic input to medial nucleus of the amygdala (MeA) neurons. MeA neurons were recorded in vitro using whole-cell recordings in the presence of $\gamma$-aminobutyric acid (GABA) blockers to isolate glutamatergic excitatory postsynaptic current (EPSC) inputs. (a) Miniature EPSCs (mEPSCs) were measured $(+\mid \mu$ tetrodotoxin (TTX)) from group-housed and isolation-reared rats. The frequency of mEPSCs was significantly less in isolation-reared rats, demonstrated in cumulative frequency plots from exemplar MeA neurons from group-housed and isolation-reared rats. (b) Social isolation decreased the frequency of mEPSC MeApd (MeA dorsal) neurons but not MeApv (MeA ventral) neurons (*⿻ $<0.05$ in post hoc Holm-Sidak's multiple comparisons test after two-way repeated-measures analysis of variance (ANOVA)). (c) The amplitude of mEPSCs was not significantly different between group-housed and isolation-reared rats, in cumulative frequency histogram (left) and grouped average data (right). (d) The ratio of the amplitude of pairs of evoked EPSCs (paired-pulse ratio, $50 \mathrm{~ms}$ interval) was measured as an index of presynaptic differences (left). There was a significant shift towards depression of excitatory synaptic input in MeApd neurons from isolation-reared rats but not MeApv neurons (* $p<0.05$ in post hoc Holm-Sidak's multiple comparisons test after two-way repeated-measures ANOVA).

after social isolation, but in a manner that depends upon the subdivision of the MeA. Presynaptic and postsynaptic factors contribute to different aspects of neuronal integration of synaptic inputs. Therefore, it is expected that presynaptic and postsynaptic abnormalities will lead to different abnormalities of synaptic integration, and would therefore lead to different impairments of synaptic integration in the MeApd and MeApv. To test whether these two types of impairments lead to different in vivo impairments, integration of synaptic inputs in MeA was measured during stimulation of LAT at 10, 20, or $40 \mathrm{~Hz}$ (Figure 4a). The ratio of the last and first evoked responses was used as an index of integration (Figure 4b; see Materials and Methods section). Synaptic integration was maximal at $20 \mathrm{~Hz}$ (integration index, main effect of frequency $p<0.0001$, $\mathrm{F}(2,93)=25.63$; integration index at $20 \mathrm{~Hz}$ significantly $>10$ and $40 \mathrm{~Hz}, p<0.05$ Holm-Sidak's multiple comparisons tests). Social isolation reduced the responsiveness of the $\mathrm{MeA}$ when measured at $10 \mathrm{~Hz}$ (control $n=16$, social isolation $n=22$ rats; social isolation $\times$ pulse number interaction $p<0.0001, \mathrm{~F}(9,324)=9.01), 20 \mathrm{~Hz}$ (control $n=15$ rats, social isolation $n=16$ rats; social isolation $\times$ pulse number interaction $p<0.0001, \mathrm{~F}(9,261)=9.77$ ), and $40 \mathrm{~Hz}$ (control $n=14$ rats, social isolation $n=16$ rats; social isolation $\times$ pulse number interaction $p=0.009, \mathrm{~F}(9,252)=2.51)$. When analyzed by the nucleus, differences in the pattern of the effects of social isolation emerged (Figure 4c, dotted line). Social isolation similarly reduced integration in MeApv across stimulation frequencies (Figure 4e; main effect of frequency $p=0.861, \mathrm{~F}(2,24)=0.1511$; frequency $\times$ pulse number interaction $p=0.328, \mathrm{~F}(18,216)=1.126$, two-way RM-ANOVA), a pattern expected with generally decreased neuronal membrane responsiveness. In contrast, the effects of social isolation on integration in the MeApd depended on the stimulation frequency (Figure $4 \mathrm{~d}$; main effect of frequency $p=0.002, \mathrm{~F}(2,24)=8.082$; frequency $\times$ pulse number interaction $p<0.0001, \mathrm{~F}(18,216)=11.62$, two-way RM-ANOVA), a pattern expected with frequency-dependent synaptic deficiency.

The MeA is still maturing during the postweaning period (Schulz et al, 2009; Cooke, 2011; De Lorme et al, 2012; Bergan et al, 2014). Social isolation can stunt the maturation of other brain regions (Sanchez et al, 1995; Helmeke et al, 2001; Lapiz et al, 2003; Gos et al, 2006; Agis-Balboa et al, 2007; Gilabert-Juan et al, 2012; Makinodan et al, 2012). The MeA abnormalities caused by social isolation may also be due to a stunting of maturation, such that $\mathrm{MeA}$ neurons display an immature phenotype. Alternatively, the abnormalities caused by social isolation may be due to an increasing impairment of function that arises over time. To test these possibilities, MeA neurons were recorded at several ages over the course of postweaning social isolation (Figure 5a). There was little evidence for postweaning developmental change in membrane responsiveness (Figure $5 \mathrm{~b}$; input resistance, main effect of age $p=0.732, \mathrm{~F}(3,95)=0.430$, one-way ANOVA; adult $n=23$ neurons per 9 rats, P22-23n=26 neurons per 8 


\section{control}

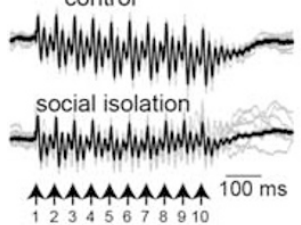

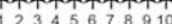

b

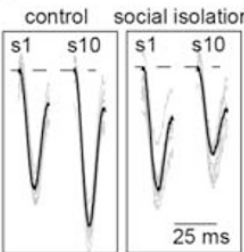

$\overline{25 \mathrm{~ms}}$

control

social isolation
C

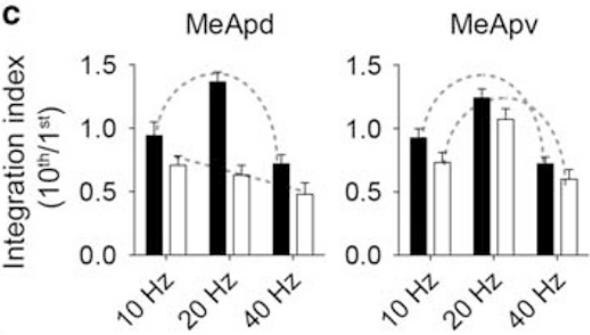

d
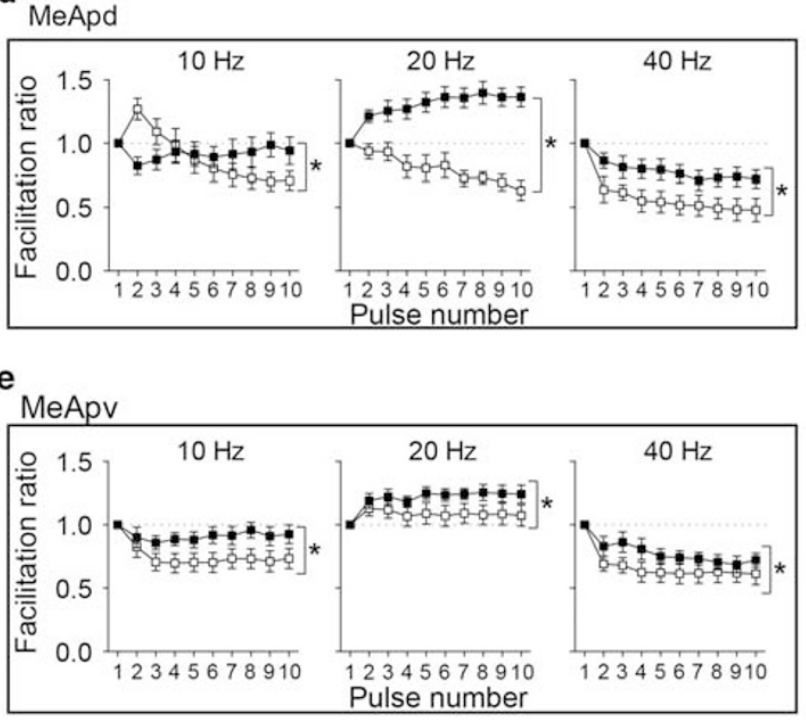
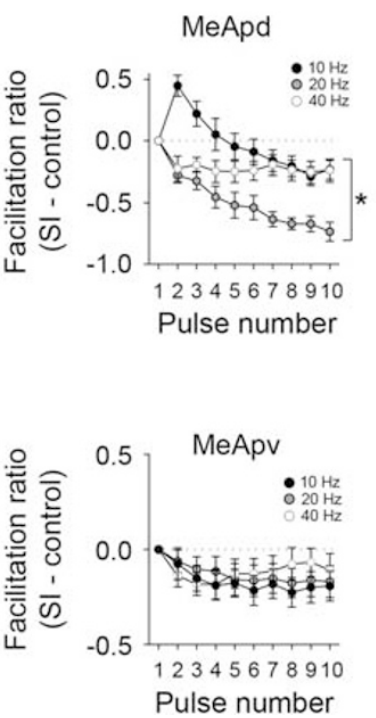

Figure 4 Postweaning social isolation impairs integration of synaptic inputs in vivo. (a) The lateral nucleus of the amygdala (LAT) was stimulated in train of I0 pulses (arrows) to evoke 10 field potentials at 10,20, or $40 \mathrm{~Hz}$. The slope of the evoked field potentials was measured. (b) An integration index can be quickly gauged by assessing the final field potential of the train (normalized to the first field potential; $\mathrm{s} \mid \mathrm{O} / \mathrm{s} \mathrm{I}$ ). A larger integration index indicates better summation of inputs. Social isolation decreases the integration index. (c) The integration index is maximal at $20 \mathrm{~Hz}$, indicative of optimal summation at this frequency. The pattern of the effects of social isolation on integration index differed between MeApd (MeA dorsal) (reduction dependent on frequency; significant social isolation $\times$ frequency interaction, two-way repeated-measures analysis of variance (RM-ANOVA)) and MeApv (MeA ventral) neurons (general reduction across frequencies; main effect of social isolation, no significant social isolation $\times$ frequency interaction, two-way RM-ANOVA). (d) The frequency-dependent effects of social isolation on integration in MeApd is verified by measuring the facilitation ratio across the stimulation trains (left; * $P<0.05$ two-way RM-ANOVA)). The effects of social isolation in MeApd (social isolation-control) is different at I0, 20, and $40 \mathrm{~Hz}$ (right; * $<<0.05$ two-way RM-ANOVA). (e) In contrast, while social isolation decreased the integration in MeApv at each frequency (* $p<0.05$ two-way RM-ANOVA), the pattern of effects of social isolation in the MeApv is similar at 10,20, and $40 \mathrm{~Hz}$ (left). The effect of social isolation in MeApv (social isolation-control) is not different at I0, 20, and $40 \mathrm{~Hz}$ (right).

rats, P28-29 $n=25$ neurons per 7 rats, P35-36 $n=25$ neurons per 7 rats) or excitability across measured ages, with only a small, not significant, shift in group-housed rats across age (Figure $5 c$; main effect of age $p=0.605$, $\mathrm{F}(3,88)=0.618$; age $\times$ current step interaction $p=0.861$, $\mathrm{F}(12,352)=0.576$, two-way RM-ANOVA; adult $n=24$ neurons per 9 rats, P22-23 $n=25$ neurons per 8 rats, P28-29 $n=19$ neurons per 7 rats, P35-36 $n=24$ neurons per 7 rats). This was further verified by measurement of $\mathrm{EC}_{50}$ and $B_{\max }$ across age in group-housed rats (Figure $5 \mathrm{~d}$ and e; $\mathrm{EC}_{50} p=0.926, \mathrm{~F}(3,80)=0.156$, one-way ANOVA; $B_{\max }$ $p=0.955, \mathrm{~F}(3,73)=0.108$, one-way ANOVA). Thus, there did not appear to be ongoing maturation of membrane excitability across this age range. In contrast, there was a clear shift in measures of presynaptic function between puberty and adulthood, with an increased frequency of mEPSCs (Figure 6a; $p=0.030, \mathrm{~F}(3,103)=3.091$, one-way ANOVA) and a shift in paired-pulse ratio (Figure $6 \mathrm{~b} ; p=0.029, \mathrm{~F}(3,55)=3.247$, one-way ANOVA). On closer examination, this maturation of excitatory input was localized to MeApd (Figure 6a and b;
mEPSC frequency, $p=0.043, \mathrm{~F}(3,56)=2.894$; paired-pulse ratio, $p=0.034, \mathrm{~F}(3,24)=3.404$, one-way ANOVA), with no significant postweaning change in presynaptic function measured in MeApv (mEPSC frequency, $p=0.374$, $\mathrm{F}(3,43)=0.374 ;$ paired-pulse ratio, $p=0.449, \quad \mathrm{~F}(3,28)=$ 0.9101, one-way ANOVA). In summary, postweaning maturation was observed only in MeApd, and it was specific for maturation of excitatory synaptic input.

Social isolation caused a progressive decrease of postsynaptic membrane responsiveness (input resistance, main effect of social isolation $p=0.041, \mathrm{~F}(1,178)=4.237$, two-way ANOVA) and a parallel decrease of membrane excitability across age, measured as $\mathrm{EC}_{50}$ (main effect of social isolation $p=0.033, \mathrm{~F}(1,163)=4.602$, two-way ANOVA). This effect of social isolation on postsynaptic neuronal input resistance and excitability was observed in MeApv neurons (Figure 5b and $\mathrm{d}$; input resistance main effect of social isolation $p=0.023, \mathrm{~F}(1,90)=5.322$, two-way ANOVA; $\mathrm{EC}_{50}$ main effect of social isolation $p=0.050, F(1,82)=3.910$, two-way ANOVA) but not MeApd neurons (Figure $5 \mathrm{~b}$ and d; input 
a
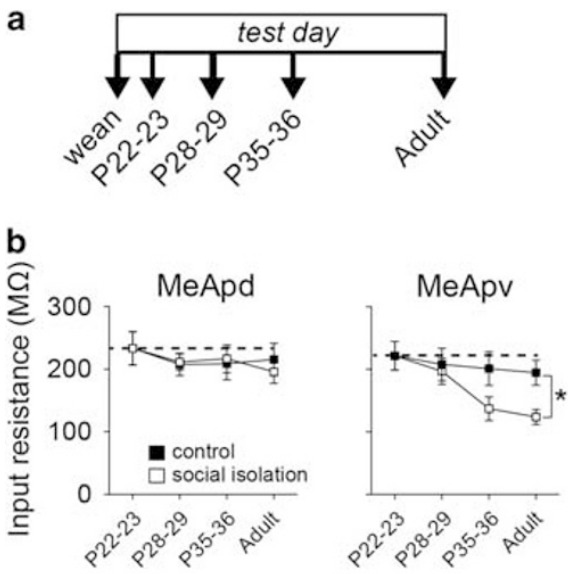

c

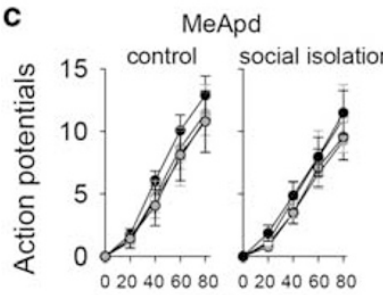

Current (pA)

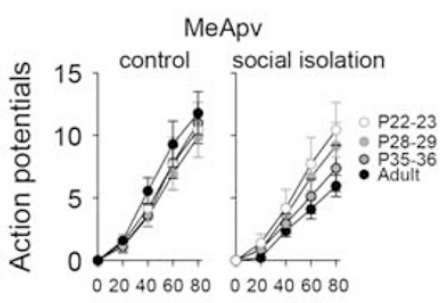

Current $(\mathrm{pA})$
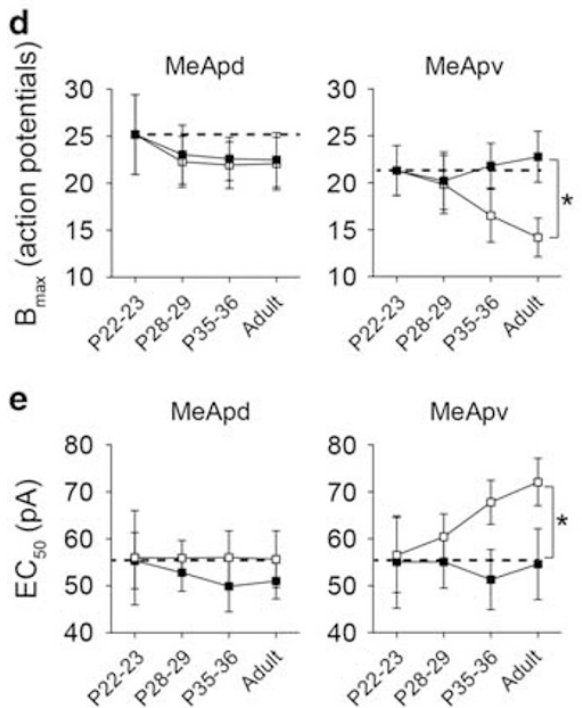

Figure 5 The time course of the isolation-rearing-induced deficits favors progressive impairment of MeApv (medial amygdala ventral) membrane excitability. (a) In vitro measures of excitability were obtained at P22-23, P28-29, P35-36, and >P50. (b) Input resistance of MeApd and MeApv neurons at different time points from weaning were similar across age in the group-housed rats. However, social isolation reduced membrane responsiveness in neurons of the MeApv but not the MeApd (medial amygdala dorsal) $(* p<0.05$ two-way repeated-measures ANOVA). (c) Membrane excitability was also measured and did not change across age in grouphoused rats. However, social isolation decreased membrane excitability in MeApv neurons, but not MeApd neurons. To facilitate comparison, membrane excitability was assessed as $B_{\max }$ (d) and $E C_{50}(e)$. There was no significant effect of age on MeA neuron excitability in group-housed rats. However, $B_{\max }$ and $\mathrm{EC}_{50}$ were both reduced in MeApv neurons from isolation-reared rats compared with group-housed rats, but not in MeApd neurons (* $p<0.05$ two-way repeated-measures ANOVA). $B_{\max }$, extrapolated maximal number of action potentials; $\mathrm{EC}_{50}$, current amplitude that evokes a $50 \%$ response. a
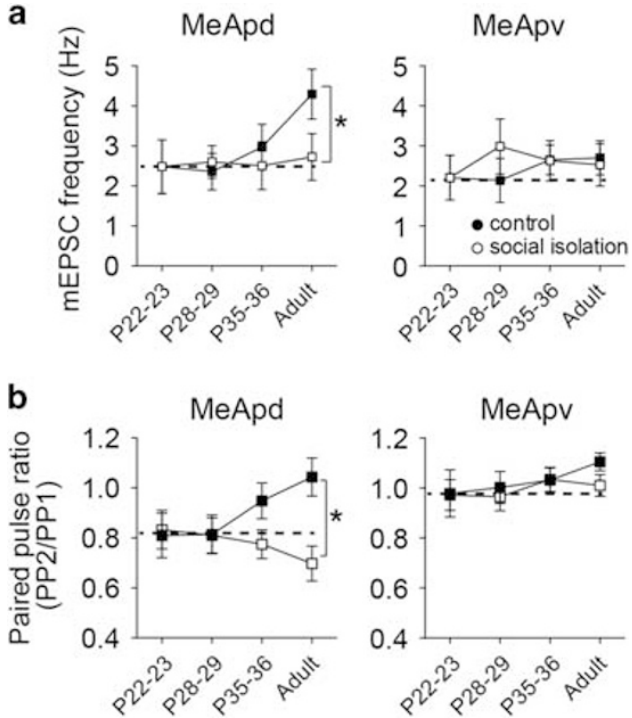

Figure 6 The time course of the isolation-rearing-induced deficits indicates stunted maturation of excitatory inputs to MeApd (medial amygdala dorsal). In vitro measures of presynaptic function were obtained at P22-23, P28-29, P35-36, and > P50. (a) There was a significant increase in excitatory input (miniature excitatory postsynaptic currents (mEPSC) frequency) to MeApd neurons across age in group-housed rats, consistent with periadolescent maturation. This increase was not observed in isolationreared rats $(* p<0.05 \mathrm{Holm}-$ Sidak's after significance in two-way analysis of variance (ANOVA)). In contrast, there was no evidence of periadolescent changes in mEPSC frequency to MeApv (medial amygdala ventral) neurons of group-housed or isolation-reared rats (right). (b) To further test the presynaptic locus of these changes, paired-pulse ratio was measured. There was a significant shift of paired-pulse ratio of excitatory input to MeApd neurons across age in group-housed rats (left), consistent with presynaptic periadolescent maturation $(* p<0.05$ two-way ANOVA). This increase was not observed in isolation-reared rats. No effect of age or isolation was observed on paired-pulse ratio of excitatory inputs to MeApv neurons (right).

resistance main effect of social isolation $p=0.907$, $\mathrm{F}(1,80)=0.0139$, two-way ANOVA; $\mathrm{EC}_{50}$ main effect of social isolation $p=0.465, \quad \mathrm{~F}(1,74)=0.5407$, two-way ANOVA). Because these postsynaptic properties did not change in control group-housed rats across the postweaning period, this effect of social isolation is consistent with a progressive impairment of postsynaptic MeApv neuronal excitability that emerges over the course of social isolation, instead of a stunting of maturation.

In contrast, while control rats displayed maturation of presynaptic function across the postweaning period, social isolation rats did not display this maturation (mEPSC frequency $p=0.899, \mathrm{~F}(3,95)=0.1954$, one-way ANOVA; paired-pulse ratio $p=0.992, \mathrm{~F}(2,39)=0.0081$, one-way ANOVA). There was a significant difference between group-housed and isolation-housed rats in presynaptic function, but this was observed only in MeApd neurons, with differences in mEPSC frequency (Figure 6a; main effect of social isolation $p=0.047, \mathrm{~F}(1,103)=4.0418$, two-way ANOVA) and paired-pulse ratio (Figure 6b; main effect of social isolation $p=0.023, \mathrm{~F}(1,48)=5.533$, two-way ANOVA). No effect of social isolation on mEPSC frequency or paired-pulse ratio was observed in MeApv neurons (Figure $6 \mathrm{~b}$ and $\mathrm{d}$; mEPSC frequency $p=0.662, \mathrm{~F}(1,87)=$ 0.1930, two-way ANOVA; paired-pulse ratio main effect of 
social isolation $p=0.403, \quad F(1,58)=0.7084$, two-way ANOVA). This indicates that a maturational stunting of excitatory drive occurs in the MeApd of rats socially isolated during the postweaning period.

\section{DISCUSSION}

The MeA has a fundamental role in a range of social behaviors. Postweaning social isolation causes impairments in many of these social behaviors. Here we found that postweaning social isolation decreased the activity of neurons in both the MeApd and MeApv. However, the underlying pathophysiology differs between these nuclei, with impaired excitatory input in the MeApd but reduced membrane excitability in the MeApv.

The postweaning period may be especially sensitive to disruptions of the social environment because social experience during this critical period is required for normal development. Postweaning social isolation within a critical preadolescent time window leads to long-term effects on social behaviors, including behaviors that rely on the MeA, such as sexual mounts/intromissions and non-contact penile erections, social aggression and avoidance, defensive response patterns during social aggression, and reduced social vocalizations (Ward and Reed, 1985; Bakker et al, 1995; Wongwitdecha and Marsden, 1996; Pellis et al, 1999; Cooke et al, 2000; Lukkes et al, 2009; Shoji and Mizoguchi, 2011; Seffer et al, 2015), and other behaviors (Hall et al, 1997, 1998; Varty and Geyer, 1998; Bakshi and Geyer, 1999; Makinodan et al, 2012). Effects of postweaning social isolation on these social behaviors may be mediated by the MeA. By analogy to other brain regions, MeA development during the postweaning period may require critical input to mature normally, such as that offered by social experience. The absence of social experience during this critical window may lead to the absence of social experience-dependent plasticity. The MeA undergoes prepubertal maturation and peripubertal refinement in response to gonadal hormones that parallels the refinement of these social behaviors (Cooke, 2006). There is enlargement of the MeApd during puberty (Romeo and Sisk, 2001; Chareyron et al, 2012; De Lorme et al, 2012) that is coupled with increased synaptophysin (Zehr et al, 2006), PSD-95, and vesicular glutamate transporter 2, consistent with increased number of excitatory synaptic inputs (Cooke, 2011). Disruption of synaptic refinement in MeApd may contribute to the effects of social isolation on social behaviors. Indeed, the normal peripubertal maturation of excitatory synaptic input, as reflected in the frequency of EPSCs and paired-pulse ratio, is stunted in the MeApd of socially reared rats (Figure 6). Further supporting this interpretation, previous findings indicate that social isolation impedes normal maturation of $\mathrm{MeA}$ volume (Cooke et al, 2000). In addition to interference with maturation, social isolation caused de novo impairments in MeApv. Progressively reduced membrane excitability of $\mathrm{MeApv}$ neurons during social isolation is expected to also contribute to behavioral impairments, and could impede the normal refinement of MeA-dependent social behaviors during development. The driving force behind these impairments caused by social isolation may be similar to long-term changes caused by sensory deprivation during critical developmental periods. Monocular deprivation and dark rearing during critical developmental windows cause transient changes followed by long-term decreases in the intrinsic and synaptic responsiveness of visual cortex (Hubel et al, 1977; Fagiolini et al, 1994; Gordon and Stryker, 1996; Smith et al, 2009; Nataraj et al, 2010). The net result of these effects of social isolation on pre- and postsynaptic function is decreased ability of MeAp neurons to become activated. This decreased responsiveness may lead to decreased guidance of social behaviors by the MeAp in adults. In particular, inputs from the basolateral amygdala, including the lateral nucleus, guide behaviors in response to conditioned social cues (Martinez et al, 2011; Takahashi et al, 2013), and inputs from olfactory regions guide social behaviors in response to pheromones and other odor cues (Kang et al, 2009; Samuelsen and Meredith, 2009a; DiBenedictis et al, 2015). Diminished responses to these inputs is expected to lead to reduced production of social behaviors (DiBenedictis et al, 2015; Noack et al, 2015). However, the outcome of these impairments on MeApd and MeApv is different, with a general decrease of responsiveness in MeApv but a more specific deficit in MeApd at higher frequency inputs. This may lead to continuous impairment of MeApv function, but impaired MeApd function only when the $\mathrm{MeA}$ is challenged by novel or complex social situations.

There are two primary types of neurons in the MeA, principle projection neurons and interneurons. Based on the degree of spike frequency adaptation, small hyperpolarization-activated voltage sag, and shape of fast afterhyperpolarization potentials, the neurons recorded in this study are most likely principle projection neurons of the MeA (Bian et al, 2008; Keshavarzi et al, 2014). Although there is a degree of overlap, many neurons of the MeApd project to the ventrolateral part of the ventral medial hypothalamus $(\mathrm{VMH})$ region important in mating behavior, whereas many neurons of the MeApv project to the dorsomedial VMH region important in agonistic behavior. Therefore, it is tempting to speculate that deficits in MeApd and MeApv function are expected to lead to inability of MeApv to direct VMH-dependent agonistic behavior, and deficiency in MeApd ability to drive VMH-dependent sexual behavior. Indeed, deficits observed after social isolation are consistent with abnormal modulation of the vigor and context of VMH-dependent sexual (Bakker et al, 1995; Cooke et al, 2000) and agonistic behavior (Potegal and Einon, 1989; Wongwitdecha and Marsden, 1996; Toth et al, 2008). Our results provide a specific neural intermediary for the effects of social isolation on the development of MeAmodulated social behavior. These results also hint at the amygdala pathology that could contribute to abnormal social behavior in those that experienced impoverished social environment during childhood.

\section{FUNDING AND DISCLOSURE}

The authors declare no conflict of interest.

\section{ACKNOWLEDGMENTS}

We thank Mallika Padival for the technical support. Grant support was provided by Simons Foundation (SFARI Award 283746 to JAR) and National Institutes of Health 
(R01MH084970 to JAR). The funding bodies had no role in the design of the study, collection, and analysis of data and decision to publish. The authors have no competing financial interests in relation to the work described.

\section{REFERENCES}

Adolphs R, Gosselin F, Buchanan TW, Tranel D, Schyns P, Damasio AR (2005). A mechanism for impaired fear recognition after amygdala damage. Nature 433: 68-72.

Adolphs R, Tranel D, Damasio H, Damasio A (1994). Impaired recognition of emotion in facial expressions following bilateral damage to the human amygdala. Nature 372: 669-672.

Agis-Balboa RC, Pinna G, Pibiri F, Kadriu B, Costa E, Guidotti A (2007). Down-regulation of neurosteroid biosynthesis in corticolimbic circuits mediates social isolation-induced behavior in mice. Proc Natl Acad Sci USA 104: 18736-18741.

Ahern M, Goodell DJ, Adams J, Bland ST (2016). Brain regional differences in social encounter-induced Fos expression in male and female rats after post-weaning social isolation. Brain Res 1630: $120-133$.

Ashwin C, Wheelwright S, Baron-Cohen S (2006). Finding a face in the crowd: testing the anger superiority effect in Asperger syndrome. Brain Cogn 61: 78-95.

Bakker J, van Ophemert J, Slob AK (1995). Postweaning housing conditions and partner preference and sexual behavior of neonatally ATD-treated male rats. Psychoneuroendocrinology 20: 299-310.

Bakshi VP, Geyer MA (1999). Ontogeny of isolation rearinginduced deficits in sensorimotor gating in rats. Physiol Behav 67: 385-392.

Baron-Cohen S, Ring HA, Wheelwright S, Bullmore ET, Brammer MJ, Simmons A et al (1999). Social intelligence in the normal and autistic brain: an fMRI study. Eur J Neurosci 11: 1891-1898.

Bergan JF, Ben-Shaul Y, Dulac C (2014). Sex-specific processing of social cues in the medial amygdala. Elife 3: e02743.

Bian X, Yanagawa Y, Chen WR, Luo M (2008). Cortical-like functional organization of the pheromone-processing circuits in the medial amygdala. J Neurophysiol 99: 77-86.

Breiter HC, Etcoff NL, Whalen PJ, Kennedy WA, Rauch SL, Buckner RL et al (1996). Response and habituation of the human amygdala during visual processing of facial expression. Neuron 17: $875-887$.

Chareyron LJ, Lavenex PB, Lavenex P (2012). Postnatal development of the amygdala: a stereological study in rats. J Comp Neurol 520: 3745-3763.

Choi GB, Dong HW, Murphy AJ, Valenzuela DM, Yancopoulos GD, Swanson LW et al (2005). Lhx6 delineates a pathway mediating innate reproductive behaviors from the amygdala to the hypothalamus. Neuron 46: 647-660.

Coccaro EF, McCloskey MS, Fitzgerald DA, Phan KL (2007). Amygdala and orbitofrontal reactivity to social threat in individuals with impulsive aggression. Biol Psychiatry 62: 168-178.

Cooke BM (2006). Steroid-dependent plasticity in the medial amygdala. Neuroscience 138: 997-1005.

Cooke BM (2011). Synaptic reorganisation of the medial amygdala during puberty. J Neuroendocrinol 23: 65-73.

Cooke BM, Chowanadisai W, Breedlove SM (2000). Post-weaning social isolation of male rats reduces the volume of the medial amygdala and leads to deficits in adult sexual behavior. Behav Brain Res 117: 107-113.

Critchley HD, Daly EM, Bullmore ET, Williams SC, Van Amelsvoort T, Robertson DM et al (2000). The functional neuroanatomy of social behaviour: changes in cerebral blood flow when people with autistic disorder process facial expressions. Brain 123: 2203-2212.
Davis FC, Johnstone T, Mazzulla EC, Oler JA, Whalen PJ (2010). Regional response differences across the human amygdaloid complex during social conditioning. Cereb Cortex 20: 612-621.

De Lorme KC, Schulz KM, Salas-Ramirez KY, Sisk CL (2012). Pubertal testosterone organizes regional volume and neuronal number within the medial amygdala of adult male Syrian hamsters. Brain Res 1460: 33-40.

DiBenedictis BT, Olugbemi AO, Baum MJ, Cherry JA (2015). DREADD-induced silencing of the medial olfactory tubercle disrupts the preference of female mice for opposite-sex chemosignals. eNeuro 2, pii: ENEURO.0078-15.2015.

Einon DF, Humphreys AP, Chivers SM, Field S, Naylor V (1981). Isolation has permanent effects upon the behavior of the rat, but not the mouse, gerbil, or guinea pig. Dev Psychobiol 14: 343-355.

Fagiolini M, Pizzorusso T, Berardi N, Domenici L, Maffei L (1994). Functional postnatal development of the rat primary visual cortex and the role of visual experience: dark rearing and monocular deprivation. Vision Res 34: 709-720.

Ferdman N, Murmu RP, Bock J, Braun K, Leshem M (2007). Weaning age, social isolation, and gender, interact to determine adult explorative and social behavior, and dendritic and spine morphology in prefrontal cortex of rats. Behav Brain Res 180: 174-182.

Fleming AS, Suh EJ, Korsmit M, Rusak B (1994). Activation of Foslike immunoreactivity in the medial preoptic area and limbic structures by maternal and social interactions in rats. Behav Neurosci 108: 724-734.

Fleming AS, Walsh C (1994). Neuropsychology of maternal behavior in the rat: c-fos expression during mother-litter interactions. Psychoneuroendocrinology 19: 429-443.

Fried I, Cameron KA, Yashar S, Fong R, Morrow JW (2002). Inhibitory and excitatory responses of single neurons in the human medial temporal lobe during recognition of faces and objects. Cereb Cortex 12: 575-584.

Fried I, MacDonald KA, Wilson CL (1997). Single neuron activity in human hippocampus and amygdala during recognition of faces and objects. Neuron 18: 753-765.

Gadziola MA, Grimsley JM, Shanbhag SJ, Wenstrup JJ (2012). A novel coding mechanism for social vocalizations in the lateral amygdala. J Neurophysiol 107: 1047-1057.

Gerall HD, Ward IL, Gerall AA (1967). Disruption of the male rat's sexual behaviour induced by social isolation. Anim Behav 15: $54-58$.

Gilabert-Juan J, Molto MD, Nacher J (2012). Post-weaning social isolation rearing influences the expression of molecules related to inhibitory neurotransmission and structural plasticity in the amygdala of adult rats. Brain Res 1448: 129-136.

Gordon JA, Stryker MP (1996). Experience-dependent plasticity of binocular responses in the primary visual cortex of the mouse. $J$ Neurosci 16: 3274-3286.

Gos T, Becker K, Bock J, Malecki U, Bogerts B, Poeggel G et al (2006). Early neonatal and postweaning social emotional deprivation interferes with the maturation of serotonergic and tyrosine hydroxylase-immunoreactive afferent fiber systems in the rodent nucleus accumbens, hippocampus and amygdala. Neuroscience 140: 811-821.

Grady CL, Keightley ML (2002). Studies of altered social cognition in neuropsychiatric disorders using functional neuroimaging. Can J Psychiatry 47: 327-336.

Hall FS, Huang S, Fong GW, Pert A, Linnoila M (1998). Effects of isolation-rearing on voluntary consumption of ethanol, sucrose and saccharin solutions in Fawn Hooded and Wistar rats. Psychopharmacology (Berl) 139: 210-216.

Hall FS, Humby T, Wilkinson LS, Robbins TW (1997). The effects of isolation-rearing of rats on behavioural responses to food and environmental novelty. Physiol Behav 62: 281-290.

Helmeke C, Ovtscharoff WJ, Poeggel G, Braun K (2001). Juvenile emotional experience alters synaptic inputs on pyramidal neurons in the anterior cingulate cortex. Cereb Cortex 11: 717-727. 
Hoffman KL, Gothard KM, Schmid MC, Logothetis NK (2007). Facial-expression and gaze-selective responses in the monkey amygdala. Curr Biol 17: 766-772.

Hol T, Van den Berg CL, Van Ree JM, Spruijt BM (1999). Isolation during the play period in infancy decreases adult social interactions in rats. Behav Brain Res 100: 91-97.

Hong W, Kim DW, Anderson DJ (2014). Antagonistic control of social versus repetitive self-grooming behaviors by separable amygdala neuronal subsets. Cell 158: 1348-1361.

Hubel DH, Wiesel TN, LeVay S (1977). Plasticity of ocular dominance columns in monkey striate cortex. Philos Trans $R$ Soc Lond Ser B 278: 377-409.

Ichikawa M, Matsuoka M, Mori Y (1993). Effect of differential rearing on synapses and soma size in rat medial amygdaloid nucleus. Synapse 13: 50-56.

Ikemoto S, Panksepp J (1992). The effects of early social isolation on the motivation for social play in juvenile rats. Dev Psychobiol 25: 261-274.

Jeon D, Kim S, Chetana M, Jo D, Ruley HE, Lin SY et al (2010). Observational fear learning involves affective pain system and Cav1.2 $\mathrm{Ca}^{2+}$ channels in ACC. Nat Neurosci 13: 482-488.

Jones AP, Laurens KR, Herba CM, Barker GJ, Viding E (2009). Amygdala hypoactivity to fearful faces in boys with conduct problems and callous-unemotional traits. Am J Psychiatry 166: 95-102.

Kang N, Baum MJ, Cherry JA (2009). A direct main olfactory bulb projection to the 'vomeronasal' amygdala in female mice selectively responds to volatile pheromones from males. Eur $J$ Neurosci 29: 624-634.

Keshavarzi S, Sullivan RK, Ianno DJ, Sah P (2014). Functional properties and projections of neurons in the medial amygdala. J Neurosci 34: 8699-8715.

Kleinhans NM, Johnson LC, Richards T, Mahurin R, Greenson J, Dawson G et al (2009). Reduced neural habituation in the amygdala and social impairments in autism spectrum disorders. Am J Psychiatry 166: 467-475.

Kleinhans NM, Richards T, Sterling L, Stegbauer KC, Mahurin R, Johnson LC et al (2008). Abnormal functional connectivity in autism spectrum disorders during face processing. Brain 131: 1000-1012.

Kollack-Walker S, Newman SW (1997). Mating-induced expression of $\mathrm{c}$-fos in the male Syrian hamster brain: role of experience, pheromones, and ejaculations. J Neurobiol 32: 481-501.

Lapiz MD, Fulford A, Muchimapura S, Mason R, Parker T, Marsden CA (2003). Influence of postweaning social isolation in the rat on brain development, conditioned behavior, and neurotransmission. Neurosci Behav Physiol 33: 13-29.

Lieberwirth C, Liu Y, Jia X, Wang Z (2012). Social isolation impairs adult neurogenesis in the limbic system and alters behaviors in female prairie voles. Horm Behav 62: 357-366.

Lukkes JL, Mokin MV, Scholl JL, Forster GL (2009). Adult rats exposed to early-life social isolation exhibit increased anxiety and conditioned fear behavior, and altered hormonal stress responses. Horm Behav 55: 248-256.

Makinodan M, Rosen KM, Ito S, Corfas G (2012). A critical period for social experience-dependent oligodendrocyte maturation and myelination. Science 337: 1357-1360.

Maras PM, Petrulis A (2010). Lesions that functionally disconnect the anterior and posterodorsal sub-regions of the medial amygdala eliminate opposite-sex odor preference in male Syrian hamsters (Mesocricetus auratus). Neuroscience 165: 1052-1062.

Marsh AA, Finger EC, Mitchell DG, Reid ME, Sims C, Kosson DS et al (2008). Reduced amygdala response to fearful expressions in children and adolescents with callous-unemotional traits and disruptive behavior disorders. Am J Psychiatry 165: 712-720.

Martinez RC, Carvalho-Netto EF, Ribeiro-Barbosa ER, Baldo MV, Canteras NS (2011). Amygdalar roles during exposure to a live predator and to a predator-associated context. Neuroscience 172: 314-328.

McDonald AJ (1996). Glutamate and aspartate immunoreactive neurons of the rat basolateral amygdala: colocalization of excitatory amino acids and projections to the limbic circuit. J Comp Neurol 365: 367-379.

Meaney MJ, Stewart J (1981). Neonatal-androgens influence the social play of prepubescent rats. Horm Behav 15: 197-213.

Melo AI, Lovic V, Gonzalez A, Madden M, Sinopoli K, Fleming AS (2006). Maternal and littermate deprivation disrupts maternal behavior and social-learning of food preference in adulthood: tactile stimulation, nest odor, and social rearing prevent these effects. Dev Psychobiol 48: 209-219.

Meredith M, Westberry JM (2004). Distinctive responses in the medial amygdala to same-species and different-species pheromones. J Neurosci 24: 5719-5725.

Nataraj K, Le Roux N, Nahmani M, Lefort S, Turrigiano G (2010). Visual deprivation suppresses L5 pyramidal neuron excitability by preventing the induction of intrinsic plasticity. Neuron 68: 750-762.

National Research Council (US) Committee for the Update of the Guide for the Care and Use of Laboratory Animals. Guide for the Care and Use of Laboratory Animals. 8th edition. National Academies Press (US): Washington (DC), 2011.

Noack J, Murau R, Engelmann M (2015). Consequences of temporary inhibition of the medial amygdala on social recognition memory performance in mice. Front Neurosci 9: 152.

Paxinos CWG (2009). By George Paxinos-The Rat Brain in Stereotaxic Coordinates. 6th edn. Elsevier Science: Amsterdam.

Pellis SM, Field EF, Whishaw IQ (1999). The development of a sexdifferentiated defensive motor pattern in rats: a possible role for juvenile experience. Dev Psychobiol 35: 156-164.

Petrulis A (2009). Neural mechanisms of individual and sexual recognition in Syrian hamsters (Mesocricetus auratus). Behav Brain Res 200: 260-267.

Phan KL, Fitzgerald DA, Nathan PJ, Tancer ME (2006). Association between amygdala hyperactivity to harsh faces and severity of social anxiety in generalized social phobia. Biol Psychiatry 59: 424-429.

Pinkham AE, Loughead J, Ruparel K, Overton E, Gur RE, Gur RC (2011). Abnormal modulation of amygdala activity in schizophrenia in response to direct- and averted-gaze threat-related facial expressions. Am J Psychiatry 168: 293-301.

Pitkanen A, Stefanacci L, Farb CR, Go GG, LeDoux JE, Amaral DG (1995). Intrinsic connections of the rat amygdaloid complex: projections originating in the lateral nucleus. J Comp Neurol 356: 288-310.

Potegal M, Einon D (1989). Aggressive behaviors in adult rats deprived of playfighting experience as juveniles. Dev Psychobiol 22: 159-172.

Richey JA, Rittenberg A, Hughes L, Damiano CR, Sabatino A, Miller S et al (2014). Common and distinct neural features of social and non-social reward processing in autism and social anxiety disorder. Soc Cogn Affect Neurosci 9: 367-377.

Rilling JK, Glenn AL, Jairam MR, Pagnoni G, Goldsmith DR, Elfenbein HA (2007). Neural correlates of social cooperation and non-cooperation as a function of psychopathy. Biol Psychiatry 61: $1260-1271$.

Romeo RD, Sisk CL (2001). Pubertal and seasonal plasticity in the amygdala. Brain Res 889: 71-77.

Rudie JD, Shehzad Z, Hernandez LM, Colich NL, Bookheimer SY, Iacoboni M (2012). Reduced functional integration and segregation of distributed neural systems underlying social and emotional information processing in autism spectrum disorders. Cereb Cortex 22: 1025-1037.

Samuelsen CL, Meredith M (2009a). The vomeronasal organ is required for the male mouse medial amygdala response to chemical-communication signals, as assessed by immediate early gene expression. Neuroscience 164: 1468-1476. 
Samuelsen CL, Meredith M (2009b). Categorization of biologically relevant chemical signals in the medial amygdala. Brain Res 1263: $33-42$.

Sanchez MM, Aguado F, Sanchez-Toscano F, Saphier D (1995). Effects of prolonged social isolation on responses of neurons in the bed nucleus of the stria terminalis, preoptic area, and hypothalamic paraventricular nucleus to stimulation of the medial amygdala. Psychoneuroendocrinology 20: 525-541.

Schulz KM, Zehr JL, Salas-Ramirez KY, Sisk CL (2009). Testosterone programs adult social behavior before and during, but not after, adolescence. Endocrinology 150: 3690-3698.

Seffer D, Rippberger H, Schwarting RK, Wohr M (2015). Pro-social $50-\mathrm{kHz}$ ultrasonic communication in rats: post-weaning but not postadolescent social isolation leads to social impairments-phenotypic rescue by re-socialization. Front Behav Neurosci 9: 102.

Shoji H, Mizoguchi K (2011). Aging-related changes in the effects of social isolation on social behavior in rats. Physiol Behav 102: $58-62$.

Smith GB, Heynen AJ, Bear MF (2009). Bidirectional synaptic mechanisms of ocular dominance plasticity in visual cortex. Philos Trans $R$ Soc Lond Ser B 364: 357-367.

Stein MB, Goldin PR, Sareen J, Zorrilla LT, Brown GG (2002). Increased amygdala activation to angry and contemptuous faces in generalized social phobia. Arch Gen Psychiatry 59: 1027-1034.

Takahashi Y, Kiyokawa Y, Kodama Y, Arata S, Takeuchi Y, Mori Y (2013). Olfactory signals mediate social buffering of conditioned fear responses in male rats. Behav Brain Res 240: 46-51.

Toth M, Halasz J, Mikics E, Barsy B, Haller J (2008). Early social deprivation induces disturbed social communication and violent aggression in adulthood. Behav Neurosci 122: 849-854.

Toth M, Tulogdi A, Biro L, Soros P, Mikics E, Haller J (2012). The neural background of hyper-emotional aggression induced by post-weaning social isolation. Behav Brain Res 233: 120-129.

Tottenham N, Hertzig ME, Gillespie-Lynch K, Gilhooly T, Millner AJ, Casey BJ (2014). Elevated amygdala response to faces and gaze aversion in autism spectrum disorder. Soc Cogn Affect Neurosci 9: 106-117.

Unger EK, Burke KJJ, Yang CF, Bender KJ, Fuller PM, Shah NM (2015). Medial amygdalar aromatase neurons regulate aggression in both sexes. Cell Rep 10: 453-462.

van den Berg CL, Hol T, Van Ree JM, Spruijt BM, Everts H, Koolhaas JM (1999a). Play is indispensable for an adequate development of coping with social challenges in the rat. Dev Psychobiol 34: 129-138.
Van den Berg CL, Pijlman FT, Koning HA, Diergaarde L, Van Ree JM, Spruijt BM (1999b). Isolation changes the incentive value of sucrose and social behaviour in juvenile and adult rats. Behav Brain Res 106: $133-142$.

Varty GB, Geyer MA (1998). Effects of isolation rearing on startle reactivity, habituation, and prepulse inhibition in male Lewis, Sprague-Dawley, and Fischer F344 rats. Behav Neurosci 112: $1450-1457$.

von dem Hagen EA, Stoyanova RS, Baron-Cohen S, Calder AJ (2013). Reduced functional connectivity within and between 'social' resting state networks in autism spectrum conditions. Soc Cogn Affect Neurosci 8: 694-701.

Von Frijtag JC, Schot M, van den Bos R, Spruijt BM (2002). Individual housing during the play period results in changed responses to and consequences of a psychosocial stress situation in rats. Dev Psychobiol 41: 58-69.

Wall VL, Fischer EK, Bland ST (2012). Isolation rearing attenuates social interaction-induced expression of immediate early gene protein products in the medial prefrontal cortex of male and female rats. Physiol Behav 107: 440-450.

Wang AT, Dapretto M, Hariri AR, Sigman M, Bookheimer SY (2004). Neural correlates of facial affect processing in children and adolescents with autism spectrum disorder. J Am Acad Child Adolesc Psychiatry 43: 481-490.

Ward IL, Reed J (1985). Prenatal stress and prepuberal social rearing conditions interact to determine sexual behavior in male rats. Behav Neurosci 99: 301-309.

Weng SJ, Carrasco M, Swartz JR, Wiggins JL, Kurapati N, Liberzon I et al (2011). Neural activation to emotional faces in adolescents with autism spectrum disorders. J Child Psychol Psychiatry 52: 296-305.

Williams LM, Das P, Harris AW, Liddell BB, Brammer MJ, Olivieri G et al (2004). Dysregulation of arousal and amygdalaprefrontal systems in paranoid schizophrenia. Am J Psychiatry 161: 480-489.

Wongwitdecha N, Marsden CA (1996). Social isolation increases aggressive behaviour and alters the effects of diazepam in the rat social interaction test. Behav Brain Res 75: 27-32.

Yusufishaq S, Rosenkranz JA (2013). Post-weaning social isolation impairs observational fear conditioning. Behav Brain Res 242: 142-149.

Zehr JL, Todd BJ, Schulz KM, McCarthy MM, Sisk CL (2006). Dendritic pruning of the medial amygdala during pubertal development of the male Syrian hamster. J Neurobiol 66: 578-590. 